\title{
Descriptive functional analysis of behavioral excesses shown by adults with Down syndrome and dementia.
}

\section{Millichap, D., Oliver, C., McQuillan, S., Kalsy, S., Hall, S. and Lloyd, V.}

\author{
Cerebra Centre for Neurodevelopmental Disorders, \\ School of Psychology, \\ University of Birmingham
}

Please use this reference when citing this work:

Millichap, D., Oliver, C., McQuillan, S., Kalsy, S., Hall, S. and Lloyd, V. (2003). Descriptive functional analysis of behavioral excesses shown by adults with Down syndrome and dementia. International Journal of Geriatric Psychiatry, 18, 844-854. 


\section{Acknowledgements}

This research was supported by a grant from the Foundation for People with Learning Disabilities.

We are grateful to the participants and there carers for their assistance in this research. 


\section{Abstract}

Objectives. The study examined the hypothesis that a functional relationship exists between social environmental events and behavioral excesses in individuals with Down syndrome and dementia.

Design. A case-series design was employed $(n=4)$ using an direct observation-based descriptive functional assessment procedure.

Methods. Observations were conducted in the natural environments of four participants over periods ranging from 11 to 15.4 hours. Data were collected on non-verbal and verbal behavioral excesses, appropriate engagement and verbal interaction with others. Social environmental events observed including both staff and peer behavior.

Results. Analysis of co-occurrence for behavioral excesses and social environmental events indicated significant relationships for some behaviours consistent with operant reinforcement processes. Sequential analysis showed that changes in the probability of social contact occurred in the period directly preceding and following verbal behaviors

Conclusions. Results support the hypothesis that, consistent with literature for older adults with dementia in the general population, some behavioral excesses were functional in nature and not randomly occurring events,. No relationship was found between appropriate engagement and staff contact. Evidence of the functional nature of target behavioral excesses indicates that behavioral interventions have potential for this client group. 


\section{$\underline{\text { Introduction }}$}

The association between Down syndrome and Alzheimer's disease has primarily been established by the presence of neuropathological signs found in those with Down syndrome over the age of 35 and may relate to the chromosomal abnormality present in most individuals (trisomy 21) (Holland \& Oliver, 1995; Oliver \& Holland, 1986). It is estimated that $37.7 \%$ of those aged over 50 years with Down syndrome develop dementia (Cooper \& Prasher, 1995). The pattern of cognitive decline in individuals with Down syndrome and dementia matches that seen in the general population (Oliver, Crayton, Holland, Hall \& Bradbury, 1998) and the pattern of behavioral signs observed in the mainstream population with Alzheimer's disease is largely found in individuals with Down syndrome. Behavioral signs of late stage dementia include urinary incontinence, loss of self care skills, reduced mobility, fatigue, wandering, uncooperative resistive behaviors, over activity and sleeplessness (Prasher \& Filer, 1995; Cooper \& Prasher, 1998).

There is little consideration in published literature regarding psychological intervention appropriate for behavioral difficulties in those with Down syndrome who develop dementia. Within the general older adult dementia literature, a number of interventions employing behavioral approaches, with or without functional analysis, are evident. Examples include interventions for deficits such as disorientation and lack of self care skills via stimulus control, backward chaining verbal and visual prompting procedures (McAvoy \& Patterson, 1986), co-morbid depression treated via increase in “pleasant” reinforcement (Teri, Logsdon, Uomoto \& McCurry, 1997), and behavioral excesses such as disruptive vocalisation via differential reinforcement and stimulus control procedures (Doyle, Zapporoni, O’Connor \& Runci, 1997). Studies employing a functional analytic methodology prior to intervention have focused on behaviors such as disruptive vocalisation (Buchanan \& Fisher, 2002, Burgio, Scilley, Hardin, Hsu \& Yancy, 1996), wandering (Heard \& Watson, 1999), agitated behavior (Moniz-Cook, Woods \& Richards, 2001; Teri et al. 1998; Bakke et al. 1994) and various 
unspecified behavioral excesses within a group design (Moniz-Cook et al. 1998). These studies lead to two conclusions. Firstly, that behavioral deficits and excesses associated with dementia can be influenced and brought under the control of environmental variables consistent with operant theory and secondly that functional analytic methods have identified environmental events that influence behavioral excesses, indicating that behaviors may be functional in nature.

There is potential overlap between work within the general dementia and mental retardation populations. The literature regarding the functional assessment and subsequent treatment of behavioral excesses within the mental retardation population is vast. A number of studies have determined the functional relationships between behavioral excesses and social lenvironmental events (e.g. Oliver and Hall, 1992; Emerson et al., 1995; 1996 and Vollmer, Borrero, Wright, Van Camp \& Lalli; 2001). Such evidence has contributed to the argument that functional assessment improves subsequent treatment efficacy (Didden, Duker \& Korzilius, 1997). However, despite evidence of the value of functional analysis within both dementia and mental retardation literatures there are no studies that have adopted this approach for behavioral excesses for people with Down syndrome and Alzheimer's disease. Consequently, it has yet to be established that the behavioral excesses those with Down syndrome and dementia show are related to environmental contingencies and thus potentially functional in nature.

The hypothesis under examination in this study was that if the behavioral excesses apparent in participants with Down syndrome and dementia were functional in nature, as would appear to be the case based on evidence from the general dementia literature, then there would be evidence of significant co-occurrence of social environmental events with behavioral excesses, although the 
nature of co-occurring events may vary in relation to possible behavioral function. This hypothesis was examined with regard to both verbal and non-verbal behavioral excesses.

\section{Method}

\section{Participants.}

As part of a larger study of Down syndrome and Alzheimer's disease, seven individuals with Down syndrome were identified as meeting criteria for a clinical diagnosis of Alzheimer's disease outlined by Holland (1999), which included the presence of gradual decline and cognitive impairment in the absence of significant health or sensory problems. Additionally, participants showed behavioral excesses in public areas, thus allowing observation to take place. This secondary inclusion criteria limited the study to four participants. Assessments included the Dementia Questionnaire for Persons with Mental Retardation (Evenhuis, Kengen \& Eurlings, 1992), the British Picture Vocabulary Scale (Dunn \& Dunn, 1997) and a semi-structured interview focusing on functional and behavioral changes was also conducted with carers The results of assessments are shown in Table 1.

\section{INSERT TABLE 1 HERE}

The Dementia Questionnaire for Persons with Mental Retardation (Evenhuis, 1992) indicated the presence of short term and long-term memory impairment, orientation difficulties, loss of practical skills, reduced activities and behavioral disturbance for Lisa. Results for Louise indicated severe deficits in social and cognitive skills. Results for Alice revealed severe deficits in cognitive and social skills. Results for Sally revealed deficits in long-term memory, orientation and mood difficulties. Lisa, Alice and Sally had a visual-impairment due to the development of cataracts. 
Behavioral excesses evident for Alice comprised of seeing "imaginary people and animals”, which had been interpreted as possible psychotic phenomena, and frequent bouts of unexplained tearfulness and crying. Stereotypic behaviors were also evident including the repeated removal and replacement of her shoes and socks. Excesses for Louise were comprised of long periods of time shouting and wandering in her environment in addition to several stereotypic behaviors, such as picking up items from the environment (e.g. pens) and holding these in her hands without any form of active manipulation. Lisa was reported to spend much of her time vocalising in a repetitive manner. Sally picked her skin and pulled out her hair and engaged in stereotypic tongue chewing and mouthing movements. All participants were reported to talk to themselves. Sally's speech to herself was sub-vocal and made up of overt irregular mouth movements consistent with speech. Lisa's speech to herself consisted of low volume mumbling and Louise's speech had deteriorated to use of single words.

\section{$\underline{\text { Procedure }}$}

Observers collected data using Hewlett Packard Jornada model 720 palm -top computers. The frequency and duration of target behaviors were recorded on OBSWIN data collection software (Martin, Oliver \& Hall, 2001). The software allows the recording of frequency and duration of target variables second by second in real-time negating the need for interval-based recording. Up to 36 variables may be recorded simultaneously. Twelve variables were recorded across all participants. These targeted behavior of participants, staff and fellow service users (peers). Participant variables were Appropriate Engagement in Activity, Talking to Others, Talking to Self, $\underline{\text { Stereotyped Behaviors, Echolalia.. Peer behaviors were Talk to Participants (Peer Talk), Peer }}$ Physical Contact (Peer Physical). Staff behaviors were Staff Verbal Demand (Staff Demand), Staff

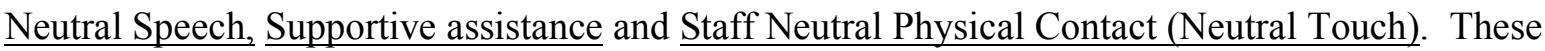


codes were devised on the basis of past observational studies of environments of adults with learning disabilities (Beasley \& Mansell, 1992; Felce et al. 1986).

A number of behavioral excesses were identified and these were operationalised during the initial semi-structured interview and formed the individual participant behavior codes. Individual behavior codes were: Imaginary contact by Alice, Groaning by Lisa, Shouting by Louise,

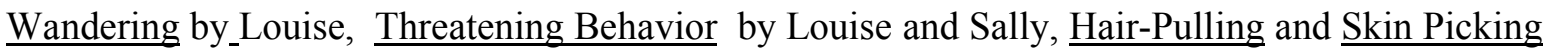
by Sally.

For three participants, observational data were gathered in-situ and for one participant (Sally), video records were obtained via a pre-positioned Sony TRV66 video camera. Observations took place in environments in which target behaviors were known to occur and were conducted during evenings, mealtimes, daytimes and weekends. For three participants, the environments were daily living areas of group homes and for the fourth (Louise), the day room and dining areas of an older adult nursing home. Observation periods were 15.4 hours (Alice), 13.9 hours (Louise) 14.1 hours (Lisa) and 11 hours (Sally).

\section{Inter Observer Reliability}

Inter-observer reliability was calculated for approximately $20 \%$ of total observations across participants. For two participants, reliability observation periods were conducted live (Lisa and Alice) and for others via video recordings. Kappa (Cohen, 1960) was calculated for each of the target variables under observation. For behavior codes used across all four participants, reliability indices were calculated on the basis of aggregated data from all participants. Where behavior codes were unique, reliabilities were calculated for each individual. Agreement was calculated based on ten-second bins for the presence of each target variable (see Oliver, Hall \& Nixon, 1999). The 
reliability of Echolalia (for all participants) and Threats by Sally were below acceptable levels $($ Kappa $<.50)$ and were omitted from subsequent analysis. The mean Kappa value for variables employed across individuals was .83 (range .58 to .98 ) and .82 for unique individual variables (range .76 to .85$)$.

\section{$\underline{\text { Data Analysis }}$}

In order to examine relationships between behaviors and environmental events, data for each observation period were appended for each participant. Datasets were analysed on the basis of tensecond windows employing Yule's Q to evaluate the magnitude of an association between a condition and a behavior or two behaviors. Yule's $\mathrm{Q}$ is calculated on the basis of a 2x2 matrix, with resultant values ranging from -1 to +1 and is an indication of the magnitude of difference between the conditional and unconditional probability of a target behavior occurring. For the purposes of analysis, a Yule's Q value of 0.5 or higher was deemed to indicate the presence of a significant association as it indicates a target-behavior is three times more likely to occur in the presence of another event (social environmental or behavior) than by chance. The comparison of conditional verses unconditional probability of behavior occurrence in identifying potential establishing operations is regarded as superior to consideration of conditional probability alone (Vollmer et al., 2001). The identification of potential function is based on the increased likelihood of a behavior occurring with certain environmental conditions (Iwata et al., 1982) as represented by the Yule's Q value. Only positive Yule's Q values were employed as staff and peer codes were exhaustive, and thus negative values are constrained, and a priori predictions were made regarding positive associations.

Yule's Q values were calculated employing the GSEQ statistical analysis package (Bakeman \& Quera, 1995). In order to undertake the calculations, target behaviors had to occur in a minimum of 
ten, ten-second windows and Throwing by Louise is absent from the analysis of co-occurrence for this reason. In order to examine the possible impact of a lack of environmental contact on the behaviors of interest, No-Contact conditions were created for both staff and peers. $\underline{\text { Staff No- }}$ Contact indicated the absence of staff Supportive Assistance, $\underline{\text { Neutral Physical, }}$ Verbal Demand and $\underline{\text { Verbal Neutral behavior. Peer No-Contact indicated the absence Peer Talk and Peer Physical. Staff }}$ $\underline{\text { contact was defined by combining Supportive assistance, }}$ Neutral Physical, Verbal Demand and $\underline{\text { Verbal Neutral behaviors. Peer contact was defined by combining Peer Talk and Peer Physical. }}$

In order to examine possible sequential relationships between behaviors, a sequential lag analysis procedure was employed. Lags were created in combined data sets for each participant on the basis of one-second intervals, for ten seconds preceding and ten seconds following the onset of participant verbal behaviors and the conditional probability of Staff and Peer Contact was calculated at each one second datum point. A period of ten seconds preceding and following the onset of each of the verbal behaviors was employed with a procedure for terminating calculation when an additional onset of verbal behaviour was identified within either 10s window.

\section{$\underline{\text { Results }}$}

Table 2 shows the durations and frequencies for participant behaviors.

\section{INSERT TABLE 2 HERE}

Rates of Talk to Self (range $1.25 \%$ to $9.25 \%$ ) were higher for all participants than Talk to Other (range 0.33 to $4.19 \%$ ) during observation periods. Stereotypic behaviors were observed in three out of four participants. For Louise, who had a more severe cognitive and functional impairment, these exceeded the percentage of time in Appropriate Engagement, which was also exceeded by 
behavioral excesses of Shouting and Wandering. For Lisa, the percentage frequency of Groaning per hour also exceeded the frequency per hour of Appropriate Engagement.

Table 3 shows the durations and frequencies for staff behaviors and indicates that the majority of staff verbal interactions with participants were demand related (range $0.85 \%$ to $3.54 \%$ ). The majority of staff physical contact is based on support (range $1.89 \%$ to $11.51 \%$ ).

\section{INSERT TABLE 3 HERE}

\section{$\underline{\text { Co-occurrence of Behaviors and Social Environmental Events }}$}

The results for the analyses of co-occurrence for verbal behaviors and sociallenvironmental events are shown in Table 4.

\section{INSERT TABLE 4 HERE}

The results in Table 4 reveal that, as might be expected for verbal behavior, for all participants, there was a significant association between participant Talk to Other and staff verbal behaviors of

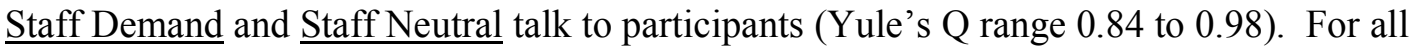

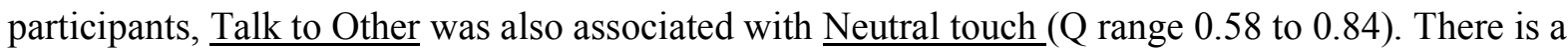
significant association between Supportive Assistance and Talk to Other for three participants (Yule's Q range 0.55 to 0.84 ). Thus, there was a relationship between verbal interaction and task related activities in these participants with the exception of Sally (Yule's $Q=0.36$ ). These results show that contact from staff is associated with participant speech. There was a significant relationship between Talk to Self and No Contact occurring from staff, i.e. no support, neutral contact or verbal interaction and Talk to Self in three participants (Yule's Q range 0.52 to 0.55 ), but not in a fourth (Louise, Yule's $Q=0.02$ ). This does not relate to staff absence from the 
environment, as coding of Talk to Self could occur in the presence of staff within the observed setting. Table 4 also reveals that Alice's occurrence of Imaginary Contact is significantly associated with $\underline{\text { Staff No Contact }}(Y u l e$ 's $Q=1.00)$. As for Talk to Self, this behavior was coded in the presence of staff within the setting. For two participants, Alice and Sally, there is a significant relationship between Talk to Other and Peer Talk $(Y u l e$ 's $Q=0.76$ and 0.92$)$, which reflects observed interaction with fellow service users. For Sally, Peer Touch is also significantly associated with $\underline{\text { Talk to Other }}($ Yule's $\mathrm{Q}=0.80$ ) indicating touch during interactions, observed in the form of $\underline{\text { Neutral Touch }}$ or Supportive Assistance, which was not discriminated in coding for peers. For one participant (Lisa), peer No Contact was associated with Talk to Other, (Yule's Q = 1.00). For Sally, Talk to Self is positively associated with peer No Contact (Yule's $Q=0.52$ ).

In summary, a significant relationship between Talk to Other and staff verbal behaviors was found. In two participants this was also the case for peer verbal behavior (Alice and Sally), although for one participant (Lisa) there would appear to be evidence of a lack of verbal interaction with peers. Talk to Others was also significantly associated with supportive staff behavior and is associated with Neutral Touch from staff. For Sally, this was also associated with physical contact from peers. Instances of Talk to Self co-occurred significantly with Staff No Contact in three participants, but not in a fourth (Louise) and Peer No Contact for Sally. Imaginary Contact by Alice is significantly associated with an absence of contact from care staff.

Table 5 shows results of the analysis of co-occurrence of social environmental events and nonverbal behavioral excesses.

INSERT TABLE 5 HERE. 
For Alice there was a significant relationship between occurrence of Stereotypy and Staff No Contact $(Y u l e$ 's $Q=0.75)$. The occurrence of Crying by Alice was associated significantly with

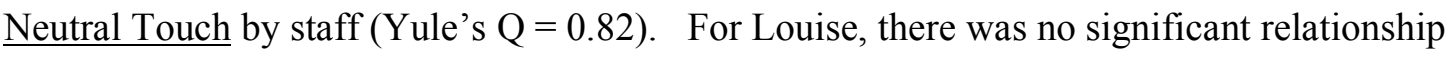
between Wandering and contact with both staff (Yule's Q range -0.64 to -0.13 ) and peers (Yule's $\mathrm{Q}=0.33)$. Threats $(Y u l e$ 's $\mathrm{Q}=0.54)$ and $\underline{\text { Shouting }}($ Yule's $\mathrm{Q}=0.70)$ are both significantly

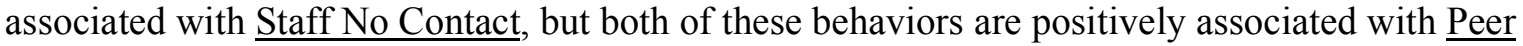
$\underline{\text { Talk. }}$ Both $\underline{\text { Threats }}(Y u l e$ 's $Q=0.54)$ and Shouting $(Y u l e ' s ~ Q=0.54)$ are positively associated with Peer Talk, indicating that these behaviors are related to Peer Contact. Crying episodes are positively associated with $\underline{\text { Staff Neutral verbal interaction (Yule's } Q=0.68)}$. Crying is also associated with Peer No Contact $(Y u l e$ 's $Q=1.00)$. Results for Lisa revealed that Groaning is associated significantly with $\underline{\text { Peer No Contact }}(Y u l e$ 's $Q=0.83)$ and no significant associations with any form of Staff Contact code (Yule's Q range -0.97 to -0.12 ). Results for Sally indicated that

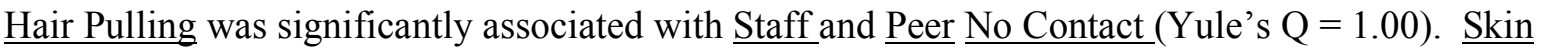

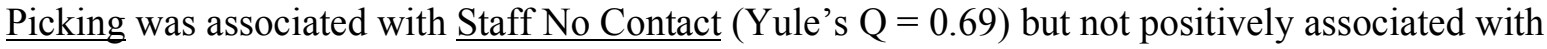
any Peer Contact behavior (Q range -0.31 to 0.37 ). Stereotypy is significantly associated with $\underline{\text { Peer }}$

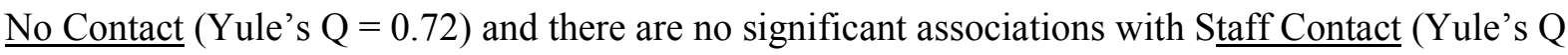

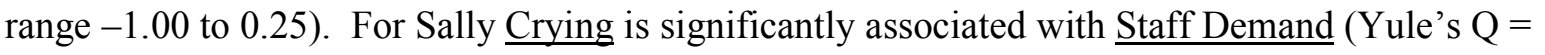
0.56) and Staff Neutral (Yule's $Q=0.51$ ) verbal interaction, and Neutral Touch (Yule's Q $=0.90)$.

In summary, stereotypic behaviors for all participants were significantly associated with no contact

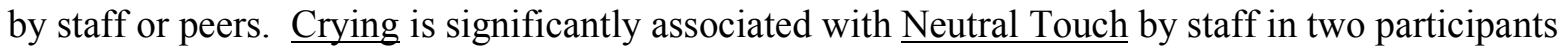
(Alice and Sally) and verbal behaviors in two (Louise and Sally). 
The evidence for co-occurrence for some verbal and nonverbal behavioral excesses indicates potential functional associations. However, sequential nature of this relationship is unclear. In order to examine sequential relationships within the data for behavioral excesses, a sequential lag analysis was employed for participant excess verbal and non-verbal behaviors. The collapsing of contact behaviors by staff and peers was undertaken in order to aid interpretation of data.

Figure 1 shows the Yule's Q values for the probability of staff and Peer Contact preceding and following verbal behavior Talk to Other and verbal behavioral excesses Talk to Self by all participants and Imaginary Contact by Alice.

\section{FIGURE 1 HERE}

As might be expected for Talk to Other the probability of $\underline{\text { Staff Contact }}$ rises in the period preceding onset for all participants and is significant for all participants. For Peer Contact the staff pattern is observed in three participants, but not in a fourth (Lisa). At onset the trend then falls before increasing again following onset for both peer and staff contact. This appears consistent with the turn taking nature of verbal interaction. For Talk to Self onset appears associated with a decline in the probability of contact from Staff and Peers, with the level of Peer Contact falling sharply in the preceding period for Lisa. The overall probability of $\underline{\text { Staff Contact appears lower than that of Talk }}$ to Other preceding and following the onset of Talk to Self. For Imaginary Contacts by Alice the probability of Staff Contact preceding and following onset is zero. However, the probability of

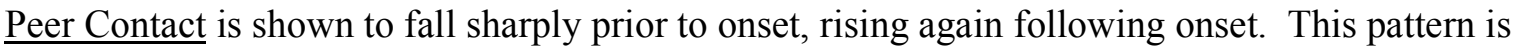
seen in Talk to Self by all participants. 
In summary the probability of $\underline{\text { Staff Contact was shown to rise prior to onset of Talk to Others. }}$ Talk to Self appeared associated with a reduced probability of contact by staff and peers. For Imaginary Contact by Alice, the pattern of Peer Contact observed for Talk to Self is apparent. Across all three forms of verbal behavior a pattern of a reduction in the probability of contact prior to the onset of the vocalisation is observed, with an increase in probability in the period following onset.

\section{$\underline{\text { Discussion }}$}

This study is novel as it employs descriptive functional analytic methodology to examine the behavioral excesses of individuals with Down syndrome and dementia. The behaviors of participants, care staff and peers were observed and recorded in real time in naturalistic environments. Behaviors observed and recorded included speech and verbal and non-verbal excesses. Recording of two behaviors, echolalia (for all participants) and threats (by Sally) failed to demonstrate adequate reliability and were not included in results. Throwing of objects by Louise did not occur frequently enough for analysis of concurrence or sequence and was withdrawn from further analysis. Statistical analyses allowed examination of concurrent and sequential relationships between behavioral excesses and social environmental events. Excess behaviors included those labelled as possible psychotic phenomena.

It was predicted that when verbal behaviors and verbal excesses occurred there would be a relationship with environmental events and that there would be evidence of sequential relationships between their occurrence and social environmental events. Data for co-occurrence shown in Table 4 demonstrate significant relationships. These include both staff contact and absence of contact, and these are observed for peers and staff behaviors. These results are consistent with a functional interpretation. That is, behaviors would not appear to be random occurrences, but related to 
environmental events. Visual inspection of trends contained in Figure 1 for sequences of staff and peer contact preceding and following onset of verbal behaviors indicated that changes in the probability of staff and peer contact occurred in the periods preceding and following onsets of behaviors. The trend observed for talking to other people by participants indicated increases in periods prior to onset, which would appear consistent with staff initiating the verbal interaction. One exception to this is found in peer contact for Lisa, which remains at zero.

Validation of the interpretation of results from this method of observation and analysis is achieved where the turn taking nature of the verbal interaction is demonstrated in the trend highlighted in the results of the sequential analysis. For the verbal excess self-talk the pattern indicates a decline in trend prior to onset and subsequent increase. Changes preceding onset are antecedent events in the form of reduced contact, which reverses following onset and is consistent with the potential reinforcement of this apparently bizarre and socially meaningless behavior. Falls in the preceding period may represent an establishing operation in the form of reduced contact. Social reinforcement of this behavior is one potential hypothesis on the basis of these data (Emerson, 2001; Vollmer, Borrero, Wright, Van Camp \& Lalli, 2001; Oliver \& Hall, 1992). Analysis of concurrent relationships and the significant relationship with no contact behavior by staff and peers for three participants (Alice, Louise \& Sally) would appear consistent with an automatic reinforcement hypothesis for this behavior (LeBlanc, Patel \& Carr, 2000). However, sequences indicate a potential social function, although a relationship with no contact or reduced contact is implicated within this. This sequence of reduced contact preceding and increased contact following onset is also observed for Alice's imaginary contacts. These results appear consistent with studies by Mace, Webb, Sharkey, Matheson \& Rosen (1988) and Mace and Lalli (1991) and the potentially functional nature of apparently bizarre speech. Mace et al. (1988) employed analogue methodology 
identifying speech as belonging to two response classes of social reinforcement and escape avoidance in an adult with a diagnosis of schizophrenia. Mace and Lalli (1991) employed a descriptive and experimental analysis of bizarre speech in a woman with mild mental retardation and a diagnosis of schizophrenia. The behavior was related to social attention occurring from peers and staff.

It was also predicted that where non-verbal behavioral excesses occurred there would be a relationship with co-occurring environmental events. Data for concurrence of non-verbal behavioral excesses are shown in Table 5 demonstrated significant relationships. These included participants' crying, wandering, shouting, threats, skin-picking, hair-pulling, stereotypy and groaning, which significantly co-occurred with contact behaviors or the absence of contact for both staff and peers. Results for hair-pulling and skin-picking exemplify the identification of potential contingencies as the observed lack of socially mediated reinforcement of these behaviors is consistent with some form of automatic reinforcement (LeBlanc, Patel \& Carr, 2000). Both hair pulling and skin-picking are associated with automatic reinforcement in many individuals (Miltenberger et al., 1998).

In focusing on specific behavioral co-occurrence and temporal sequences the results obtained in this study go beyond examples of descriptive functional assessment available in the general older adult dementia literature, which have focused on factors such as temporal and physical location of behaviors and inferred function on this basis (Burgio et al., 2001; Burgio et al., 1994). The relationships between participant behavior and social environmental events identified in this study are clear evidence for the potential operation of environmental contingencies on the occurrence of behavioral excesses and deficits consistent with an operant formulation. This would appear to 
contradict purely neurologically based hypotheses of behavioral excess or deficit. However, there is a growing acceptance of a potential interrelationship between environmental operants, neurological and genetic factors in a number of behavioral excesses including over-eating in PraderWilli Syndrome (Kennedy, Caruso \& Thomson, 2001) and self-injurious behavior in Rett Syndrome (Oliver, Murphy, Crayton \& Corbett, 1993). Within the general older adult literature related to behavioral excesses of dementia there is still a paucity of studies employing functional analytic methodology to examine difficulties associated with ageing such as dementia despite growing recognition of utility (Burgio \& Lewis, 1999). This contrasts with the literature in mental retardation, where descriptive functional assessment is recognised as a clinically valid method of inferring behavioral function (Emerson 2001) with many published examples examining a variety of behavioral excesses (For example, Vollmer et al 2000; Emerson Thomson, Reeves, Henderson, \& Robertson, 1995; Emerson, Reeves, Thomson, Henderson \& Robertson, 1996; Oliver \& Hall, 1992).

Although the results of the present study indicate descriptive analysis as a potentially useful method of examining behavioral excesses and deficits with the population of people with Down syndrome and dementia, a number of limitations are evident. Behavioral excesses are widespread amongst the general mental retardation population with prevalence increasing with severity (Emerson, 2001). The adaptive functioning of individuals with Down syndrome is extremely varied, as is cognitive functioning (Oliver \& Holland, 1986). The short-term nature of the present study may mean that the behavioral excesses and deficits examined do not relate to dementia. The longest informant relationship was three years. This is of particular relevance to self-talk as a recent study identified this behavior as possibly widespread in young adults with Down syndrome (Glenn \& Cunningham, 2001). Results of the study by Glenn and Cunningham (2001) indicated a high proportion of their 
sample talked to themselves (91\%) and the remainder, with one exception, had done so at some stage in the past. This behavior was not associated with pathology evidenced by behavioral difficulty or psychiatric diagnosis.

A further confound is the diagnosis of cataracts in study participants. A relationship between reduced visual acuity and cognitive impairment and apparent occurrence of visual hallucinations in those with Alzheimer's disease has been identified in the general population (Chapman, Dickinson, McKeith, \& Ballard, 1999). It may be that talking to self and imaginary contacts are related to this phenomenon. A further potential confound is the limited variety of activities that made up the appropriate engagement category. For three participants, eating and drinking independently made up a large part of the behavior coded, with prolonged periods spent in meals due to latencies (in one case 45 minutes to drink). A further modification for any future study might be inclusion of temporal information, as employed in studies by Burgio, to enable examination of factors implicated as having an impact on behavioral excesses in the general population with dementia such as circadian rhythm (Burgio et al, 1994). The validity of the results of the present study might also be strengthened by the application of an experimental analogue phase as has been employed elsewhere, such as in the study of Emerson et al. (1995).

The present study has a number of implications for future research and clinical intervention within the population of people with Down syndrome and dementia. The technology of functional assessment is readily available within general mental retardation services. Clinicians are trained in its application and there are a number of potential interventions available with studies indicating efficacy for the response classes of automatic and social reinforcement outlined in the present study. In addition, a wide body of research studies within the general population with dementia diagnosis 
focusing on the application of functional assessment and intervention and behavioral techniques employing management of contingent and antecedent relationships is available. The apparent relationship between behavioral occurrence and environmental events highlights the potential utility of these approaches in the Down syndrome population with dementia diagnosis. These include interventions focusing on behavioral deficits in addition to excesses. A further implication of the results of this study is the role of peers in reinforcing behavioral excesses, where descriptive and analogue based functional assessment has focused on staff and carers as mediators of reinforcement of behavioral excesses (for example, the studies by Emerson, 1995 and Vollmer et al. 2001). An important variable is perhaps being neglected in current staff and carer focused research strategies. 


\section{$\underline{\text { References }}$}

Bakeman, R., \& Quera, V. (1995). Analyzing Interaction: Sequential Analysis with SDIS and GSEQ. Cambridge University Press: Cambridge.

Bakke, B.L., Kuale, S., Burns, T., Riley McCarten, J., Wilson, L., Maddex, M., et al., (1994). Multi component intervention for agitated behavior in a person with Alzheimer;s disease. Journal of Applied Behavior Analysis, 27, 175-176.

Baltes, M.M. (1988). The etiology and maintenance of dependency in the elderly: Three phases of operant research. Behavior Therapy, 19, 301-319.

Baltes, M.M. (1996). The many faces of dependency in old age. Cambridge: Cambridge University Press.

Baltes, P.B., \& Baltes, M.M. (1990). Psychological perspectives on successful aging: The model of selective optimisation with compensation. In P.B. Baltes \& M.M. Baltes (Eds), Successful Aging:Perspectives from the Behavioral Sciences (pp1-34). Cambridge: Cambridge University Press.

Baltes, M.M., Burgess, R.L., \& Stewart, R. (1980). Independence and dependence in self care behaviors in Nursing home residents: An operant observational study. International Journal of Behavioral Development, 3, 489-500.

Baltes, M.M., Honns, S., Barton, E.M., Orzech, M.J., \& Logo, D. (1983). On the social ecology of dependence and independence in nursing home residents: A replication and extension. Journal of Gerontolgy, 38, 556-564.

Baltes, M.M., Kinderman, T., Reisenzein, R., \& Schmid, U. (1987). Further observational data on the behavioral and social world of institutions for the aged. Psychology \& Aging, 2, 390-403. 
Baltes, M.M., Neumann, E.M. \& Zank, S. (1994). Maintenance and rehabilitation of independence in old age: An intervention program for staff. Psychology and Aging, 9, 179-188.

Baltes, M.M., \& Wahl, H. -W. (1992). The dependency support script in institutions: Generalisation to community settings. Psychology \& Aging, 7, 409-418.

Buchanan, J.A., \& Fisher, J.E. (2002). Functional assessment and non-contingent reinforcement in the treatment of disruptive vocalisation in elderly dementia patients. Journal of Applied Behavior Analysis, 35, 99-103.

Burgio, L.D., \& Lewis, T. (1999). Gerontology and functional analysis, In, Functional Analysis of Problem behaviour; From effective assessment to effective support (pp305-320). Wadsworth: London.

Burgio, L.D., Scilley, K., Hardin, M., \& Hsu, C. (2001). Temporal patterns of disruptive vocalization in elderly nursing home residents. International Journal of Geriatric Psychiatry, 16, 378-386.

Burgio, L.D., Scilley, K., Hardin, M., Hsu, C. \& Yancey, J. (1996). Environmental "white noise”: An intervention for verbally agitated nursing home residents. Journal of Gerontology, 51B, 364373.

Burgio, L.D., Scilley, K., Hardin, M., Janoosky, J., Bonino, P., Slater, S.C., et al. (1994). Studying disruptive vocalization and contextual factors in the Nursing home using computer-assisted real time observation. Journal of Gerontology: Psychological Sciences, 49, 230-239.

Cohen, J. (1960). A coefficient of agreement for nominal scales. Educational and Psychological Measurement, 20, 37-46.

Cooper, S.A., \& Prasher, V.P. (1998) Maladaptive behaviours and symptoms of dementia in adults with Down's syndrome compared with adults with intellectual disability of other aetiologies. Journal of Intellectual Disability Research, 42 , 293-300. 
Didden, R., Duker, P. C., \& Korzilius, H. (1997). Meta-analytic study on treatment effectiveness for problem behaviors with individuals who have mental retardation. American Journal on Mental Retardation, 101, 387-399.

Doyle, C., Zapporoni, T., O’Connor, D. \& Runci, S. (1997). Efficacy of psychosocial treatments for noisemaking in severe dementia. International Psychogeriatrics, 9, 405-422.

Dunn, L., \& Dunn, L. (1997). The British Picture Vocabulary Scale. London: NFER-Nelson.

Emerson, E. (2001). Challenging behaviour: Analysis and intervention in people with severe intellectual Disabilities. Cambridge: Cambridge University Press.

Emerson, E., Beasley, F., Offord, G., \& Mansell, J. (1992). An evaluation of hospital based specialized staffed housing for people with seriously challenging behaviours. Journal of Intellectual Disability Research, 36, 291-307.

Emerson, E., Thomson, S., Reeves, D., Henderson, D., \& Robertson, J. (1995) Descriptive Analysis of Multiple Response Topographies of Challenging Behavior across two settings. Research in Developmental Disabilities, 16, 301-329.

Evenhuis, H.M., Kengen, M.M.F., \& Eurlings, H.A.L. (1992). Evaluation of a screening instrument for dementia in ageing mentally retarded persons. Journal of Intellectual Disability Research, 36, $337-347$

Felce, D., deKock, U., \& Repp, A.C. (1986). An eco-behavioral analysis of small communitybased houses and traditional large hospitals for severely and profoundly mentally handicapped adults. Applied Research In Mental Retardation, 7, 393-408.

Felce, D., Saxby, H., deKock, U., Repp, A.C., Agar, A., \& Blunden, R. (1987). To what behaviors do attending adults respond? A replication. American Journal of Mental Deficiency, 91, 496-504. 
Glenn, S.M., \& Cunningham, C.C. (2001). Parents' reports of young people with Down syndrome talking out loud to themselves. Mental Retardation, 38, 498-505.

Heard, K. \& Watson, T.S. (1999). Reducing wandering by persons with dementia using differential reinforcement. Journal of Applied Behavior Analysis, 32, 381-384.

Holland, A.J. \& Oliver, C. (1995). Down's syndrome and the links with Alzheimer's disease. Journal of Neurology, Neurosurgery, and Psychiatry, 59, 111-114.

Hussain, R.A. (1988). Modifications of behaviors in dementia via stimulus manipulation. Clinical Gerontologist, 8, 37-43.

Hussain, R.A. \& Brown, D.C. (1987). Use of two-dimensional grid patterns to limit hazardous ambulation in demented patients. Journal of Gerontology, 42, 558-560.

Iwata, B., Dorsey, M.F., Slifer, K.J., Bauman, K.E., \& Richman, G.S. (1982). Toward a functional analysis of self-injury. Analysis \& Intervention in Developmental Disabilities, 2, 3-20.

Kindermann, T. (1993). Fostering independence in mother-child interaction: Longitudinal changes in Contingency patterns as children grow competent in developmental tasks. International Journal of Behavioral Development, 16, 513-535.

Kennedy, C.H., Caruso, M., \& Thomson, T. (2001). Experimental analysis of gene-brain-behavior relationships: some notes on their application. Journal of Applied Behavior Analysis, 34, 539-549.

LeBlanc, L.A., Patel, M.R., \& Carr, J.E. (2000). Recent advances in the assessment of aberrant behavior maintained by automatic reinforcement in individuals with developmental disabilities, Journal of Behavior Therapy and Experimental Psychiatry, 31, 137-154.

Mace, C.F., \& Lalli, J.S. (1991). Linking descriptive and experimental analysis in the treatment of bizarre speech. Journal of Applied Behavior Analysis, 24, 553-562. 
Mace, C.F., Webb, M.E., Sharkey, R.W., Mattson, D.M., \& Rosen, H.W. (1988). Functional analysis and treatment of bizarre speech. Journal of Behavior Therapy and Experimental Psychiatry, 19, 289-296.

Mansell, J., \& Beasley, F. (1993). Small staffed houses for people with a severe learning disability and challenging behaviour. British Journal of Social Work, 23, 329-344.

McEvoy, C.L., \& Patterson, R.L. (1986). Behavioral treatment of deficit skills in dementia patients. The Gerontologist, 26, 475-478.

Miltenberger, R.G., Long, E.S., Rapp, J.T., Lumley, V.A., \& Elliot, A.J. (1998). Evaluating the function of hair pulling. Behavior Therapy, 29, 211-210.

Moniz-Cook, E., Agar, S., Silver, M., Woods, R., Wang, M., Elston, C. et al. (1998). Can staff training reduce behavioral problems in residential care for the elderly mentally ill? International Journal of Geriatric Psychiatry, 13, 149-158.

Moniz-Cook, E., Woods, R.T. \& Richards, K. (2001). Functional analysis of challenging behavior in Dementia. International Journal of Geriatric Psychiatry, 16, 45-56.

Oliver, C., Crayton, L., Holland, A.J., Hall, S., \& Bradbury, J. (1998) A four year retrospective study of age-related cognitive changes in adults with Down's syndrome. Psychological Medicine, $28,1365-1377$.

Oliver, C., \& Hall, S. (1992). Differential effects of severe self-injurious behaviour on the behaviour of others. Behavioural Psychotherapy, 20, 355-365.

Oliver, C., Hall, S., \& Nixon, J. (1999). The molecular and molar analysis of communicative and problem behaviors. Research in Developmental Disabilities, 20, 197-213.

Oliver, C., \& Holland, A.J. (1986) Down's syndrome and Alzheimer's disease: A review. Psychological Medicine, 16, 307-322. 
Oliver, C., Murphy, G., Crayton, L., \& Corbett, J. (1993). Self-injurious behavior in Rett syndrome: Interactions between features of Rett syndrome and operant conditioning. Journal of Autism and Developmental Disorders, 23, 91-109.

Prasher, V.P. (1997). Dementia questionnaire for persons with mental retardation (DMR): Modified criteria for adults with down's syndrome. Journal of Applied Research in Intellectual Disabilities, $10(1), 54-60$.

Prasher, V.P., \& Filer, A. (1995) Behavioural Disturbance in People with Down's Syndrome and Dementia. Journal of Intellectual Disability Research, 39 , 432-436.

Sparrow, S., Balla, D., \& Cicchetti, V. (1994). Vineland Adaptive Behavior Scales (Interview Edition Expanded Form). American Guidance Services.

Teri, L., Logson, R.G., Uomoto, J., \& McCurry, S.M. (1997). Behavioral treatment of depression in dementia patients: A controlled clinical trial. Journal of Gerontology: Psychological Sciences, 52B, $159-166$.

Teri, L, Logson, R.G., Whall, A.L., Weiner, M.F., Trimmer, C., Perskind, E. et al (1998). Treatment for agitation in dementia patients: A behavior management approach. Psychotherapy, 35(4), 436443.

Thomas, M., Felce, D., deKock, U., Saxby, H., \& Repp, A. (1986). The activity of staff and of severely and profoundly mentally handicapped adults in residential settings of different sizes. British Journal of Mental Subnormality, 32, 82-92.

Vollmer, T., Borrero, J.C., Wright, C.S, Van Camp, C., \& Lalli, J.S. (2001). Identifying possible contingencies during descriptive analysis of severe behavior disorders. Journal of Applied Behavior Analysis, 34, 269-287. 